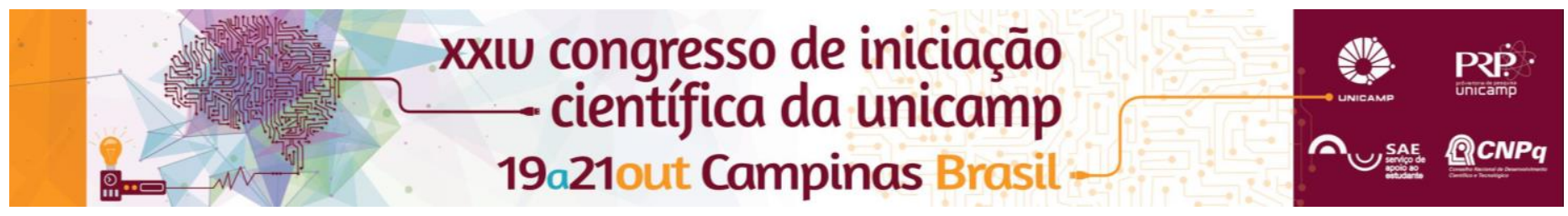

\title{
Investigação do Infiltrado Inflamatório Tumoral no Câncer de Mama e sua Relação com Parâmetros do Sangue Periférico e Adipocinas.
}

\author{
Eric E. Kodama (IC)1, Luciana Regina Moreira (PQ)2, Aline B. Santana (PG)1, Maria Salete C. Gurgel (PQ)2,3, \\ Sílvia de Barros-Mazon (PQ)1. 1Departamento de Patologia Clínica, 2CAISM-Hospital da Mulher J. A. Pinotti e \\ 3Departamento de Tocoginecologia, Faculdade de Ciências Médicas, Unicamp.
}

\section{Resumo}

Esse trabalho investigou o tipo e a intensidade de infiltrado inflamatório tumoral no câncer mamário e suas relações com os neutrófilos, linfócitos e razão NLR do sangue periférico, bem como com as concentrações séricas das adipocinas SAA e APN.

Palavras-chave: câncer de mama, obesidade e resposta inflamatória.

\section{Introdução}

A obesidade é considerada um fator de risco para o câncer mamário e cursa com estado inflamatório subclínico, com aumento de secreção de adipocinas inflamatórias, como a soro amilóide $A$ (SAA), e a diminuição de produção de adiponectina (APN) pelo tecido adiposo. Concentrações séricas elevadas de SAA e diminuídas de APN estão relacionadas com 0 desenvolvimento e/ou prognóstico do câncer de mama.1,2 Adicionalmente, os neutrófilos vêm sendo associados ao pior prognóstico de vários cânceres, inclusive 0 de mama. Mais recentemente, o marcador inflamatório, Razão Neutrófilo/Linfócito $(\mathrm{NLR})^{3}$ e a pesquisa do infiltrado inflamatório tumoral ${ }^{4}$ têm recebido destaque como potenciais fatores prognósticos neste tipo de câncer. Pelo nosso conhecimento, até o momento não existe relato na literatura de investigação das relações entre infiltrados tumorais e parâmetros do sangue periférico no câncer mamário. Desta forma, este estudo teve como objetivo a avaliação do tipo de infiltrado inflamatório tumoral e suas relações com as quantificações de neutrófilos, linfócitos, NLR no sangue periférico e com as concentrações séricas de SAA e APN, em pacientes com câncer de mama.

\section{Resultados e Discussão}

A análise do infiltrado inflamatório foi realizada em lâminas resultantes das peças cirúrgicas submetidas a exame anátomo-patológico, provenientes de 115 pacientes com carcinoma ductal invasivo, que aceitaram participar deste estudo. Após leitura e assinatura do TCLE e antes do procedimento cirúrgico, foram obtidas medidas antropométricas das pacientes, bem como amostras de sangue para avaliação laboratorial. As regiões teciduais de análise nas lâminas coradas com HE foram: Tecido Tumoral Epitelial (TEp), Tumoral Estromal (TEs), Não Tumoral (NT), Gordura Próxima (GP) e Gordura Distante (GD) do tumor. Foram avaliados o tipo e a intensidade dos infiltrados. O tipo foi classificado em mononuclear $(M)$, polimorfonuclear $(P)$, ausente (A) e as versões mistas, com predomínio respectivo de um ou de outro, "MM" ou "MP". A intensidade foi graduada em $1=$ discreta $(\leq 5 \%), 2=$ leve $(>5 \leq 20 \%), \quad 3=$ moderada $(>20 \leq 70 \%)$ e $4=a c e n t u a d a$ $(>70 \%)$. Conforme se verifica na tabela 1 , nos tecidos epiteliais TEp e NT os infiltrados detectados foram exclusivamente do tipo $M$, enquanto que no tecido estromal tumoral (TEs), além do infiltrado $M$, também se observou a presença de infiltrado misto (MM) em $12 \%$ das lâminas examinadas, que eram provenientes na sua maioria (79\%), de portadoras de câncer mamário com sobrepeso/obesidade (SP/O). Nos tecidos GP e GD a presença de infiltrado $\mathrm{M}$ foi observada respectivamente em apenas $10 \%$ e $1 \%$ das lâminas, sendo $75 \%$ provenientes de pacientes com SP/O. Para avaliação das relações dos infiltrados inflamatórios teciduais com as variáveis do sangue periférico de interesse, as pacientes foram classificadas de acordo com os graus de intensidade dos infiltrados em grupos 1, 2 e 3/4 (dependendo do tecido analisado). Maiores valores de NLR $(p=0,0086)$ foram observados entre as pacientes agrupadas sob o grau de intensidade 2 dos infiltrados no TEs. Não foram observadas associações com as outras variáveis do sangue periférico, no TEs ou nos demais tecidos analisados.

Tabela 1. Frequências dos tipos e intensidades dos infiltrados inflamatórios nas diferentes regiões teciduais analisadas.

\begin{tabular}{cccccc}
\hline & $\begin{array}{c}\boldsymbol{E p} \\
(\mathrm{n}=114)\end{array}$ & $\begin{array}{c}\text { Es } \\
(\mathrm{n}=114)\end{array}$ & $\begin{array}{c}\boldsymbol{N T} \\
(\mathrm{n}=113)\end{array}$ & $\mathbf{G P}$ & $\mathbf{G D}$ \\
$(\mathrm{n}=114)$ & $(\mathrm{n}=115)$ \\
\hline Tipo (\%) & & & & & \\
M & 100 & 88 & 57 & 10 & 1 \\
P & 0 & 0 & 0 & 0 & 0 \\
MM & 0 & 12 & 0 & 0 & 0 \\
MP & 0 & 0 & 0 & 1 & 0 \\
A & 0 & 0 & 43 & 89 & 99 \\
Intensidade (\%) & & & & & \\
Discreta (1) & 46 & 10 & 39 & 4 & 1 \\
Leve (2) & 44 & 53 & 18 & 5 & 0 \\
Moderada (3) & 10 & 34 & 0 & 1 & 0 \\
Acentuada (4) & 0 & 3 & 0 & 0 & 0 \\
Ausente (0) & 0 & 0 & 43 & 90 & 99 \\
\hline
\end{tabular}

\section{Conclusões}

Infiltrados inflamatórios de intensidade leve no tecido estromal tumoral (TEs) guardaram relação com valores de NLR mais elevados no sangue periférico. Infiltrados MM e $M$, respectivamente nos tecidos TEs e GP, foram mais frequentes em lâminas de portadoras de câncer mamário, com sobrepeso/obesidade.

\section{Agradecimentos}

\section{FAPESP, PIBIC/CNPq and FAEPEX-Unicamp.}

${ }^{1}$ Santana AB, Gurgel MSC, Montanari JFO, Bonini FM, de Barros-Mazon S. Cance Epidemiol Biomarkers Prev;. 2013; 22(2):270-4.

${ }^{2}$ Mantzoros C, Petridous E, Dessyptis N, Chavelas C, Dalamaga M, Alexe DM, et al. J Clin Endocrinol Metab. 2004; 89:1102-7.

${ }^{3}$ Azab B, Bhatt VR, Phookan J, Murukutla S, Kohn N, Terjanian, T et al. Ann Surg Oncol. 2012;19(1):217-224.

${ }^{4}$ Salgado R, Darkent C, Demaria S, et al. Annals of oncology, 2015;26(2):259-271. 